\title{
Crossing the seven Cs of internal consistency: Assessing the reliability of formative instruments
}

Stefan L. K. Gruijters, Bram P. I. Fleuren \& Gjalt-Jorn Ygram Peters

This document is the full text of "Crossing the seven Cs of internal consistency: Assessing the reliability of formative instruments", a manuscript that has been submitted for publication.

Please cite this document as:

Gruijters, S. L. K., Fleuren, B. P. I. \& Peters, G.-J. Y.(202I). Crossing the seven Cs of internal consistency: Assessing the reliability of formative instruments [version I]. PsyArXiv. https://doi.org/IO.31234/osf.io/qar39

Note that this version of the manuscript that lies before you may be outdated. The most current version of this manuscript is hosted at PsyArXiv at https://psyarxiv.com/qar39 - where a link to the final DOI will also be available once the manuscript has been accepted. Therefore, please always check that URL before citing this article! 


\title{
ASSESSING THE RELIABILITY OF FORMATIVE INSTRUMENTS
}

Running head: Assessing the reliability of formative instruments

Crossing the seven Cs of internal consistency: Assessing the reliability of formative instruments

\author{
Stefan L.K Gruijters ${ }^{\text {a }}$ \\ Open University of the Netherlands \\ Bram P.I. Fleuren ${ }^{b}$ \\ Maastricht University \\ Gjalt-Jorn Y. Peters ${ }^{b, c}$ \\ Open University of the Netherlands \\ Maastricht University
}

Author note

${ }^{a}$ General Psychology, Faculty of Psychology, Open University of the Netherlands, Heerlen, the Netherlands

${ }^{\mathrm{b}}$ Work and Social Psychology, Faculty of Psychology and Neuroscience, Maastricht University, Maastricht, the Netherlands.

${ }^{\mathrm{c}}$ Methodology \& Statistics, Faculty of Psychology, Open University of the Netherlands, Heerlen, the Netherlands

Correspondence concerning this article should be addressed to Stefan Gruijters, Faculty of Psychology and Educational Science, Valkenburgerweg 177, 6419 AT, Heerlen, The Netherlands. E-mail: mail@stefangruijters.nl. All scripts, functions and other materials described are publicly available at https://osf.io/7a95b/?view only=abc5f0dc54b4476d8890ee9f6f0d2fe4 


\begin{abstract}
Evaluating the reliability of measurement instruments is common practice in organizational psychology. When measurement proceeds using composites of multiple indicators, reliability is often examined with internal consistency estimates. This is common practice for both measurement instruments with a reflective measurement model, where a latent psychological construct is assumed to cause the item scores, and with a formative measurement model, where the composite score defines the construct. In the latter case, however, internal consistency indices such as Coefficient Alpha or Omega are not informative about reliability. In this paper, we review the assumption of reflective measurement and discuss why internal consistency estimates assume this model. We then provide an illustration of a checklist (originally proposed by Jarvis et al., 2003) intended to aid researchers, reviewers and editors in recognizing reflective measurement. Finally, the paper describes how researchers can estimate the reliability of instruments that use formative items. With these contributions, the paper aims to promote awareness of measurement assumptions when estimating reliability, and to provide tailored alternatives for assessing the reliability of formative instruments.
\end{abstract}




\section{Crossing the seven Cs of internal consistency}

Psychological tests remain ubiquitous in the organizational psychology literature.

Consequentially, the validity and reliability of these tests are important targets of empirical investigation. Organizational psychologists use various psychometric techniques to get a sense of the quality of their measurements. Common tactics include the use of factor analysis and estimating reliability using internal consistency indices - though more extensive psychometric protocols beyond factor and reliability analysis may be warranted (see Dima, 2018; Hussey \& Hughes, 2020; Maul, 2017). Estimating, evaluating, and curtailing measurement error is critical to the quality of statistical inference (Loken \& Gelman, 2017; Schmidt \& Hunter, 1999). However, whether a specific psychometric procedure provides an inroad towards estimating reliability hinges on the measurement model of an instrument.

Measurement models encountered in the measurement literature include the reflective, formative, and network models (Bollen \& Bauldry, 2011; Borsboom et al., 2003; Borsboom \& Cramer, 2013; Fleuren et al., 2018; Gruijters \& Fleuren, 2018; Jarvis et al., 2003). Reflective models position the theoretical construct as causally responsible for variance on indicators - the way that the g-factor was initially proposed to account for the positive manifold on IQ indicators. Conversely, formative models assume a construct to be formed or created by the variance on its indicators, so that different indicators (e.g., education background, income, and ZIP code) can be used to create an index of the construct of interest (e.g., socioeconomic status). Network models represent a construct in terms of a (causally undirected) network of associated indicators (Borsboom \& Cramer, 2013). Network models have been argued to apply to complex constructs that are not reasonably captured as a single variable, such as major depressive disorder, and other psychopathology (Borsboom et al., 2011; Borsboom \& Cramer, 2013; Fried et al., 2017; Fried \& Nesse, 2015).

Overall, we distinguish three purposes of reflective, formative and network modelsrespectively, to measure an attribute, to create an instrumental index, or to represent a construct (Fried, 2017; McGrath, 2005). Which of these goals is reasonably pursued by researchers often depends on the indicators that are used, and how they are thought to relate to the measured construct (Borsboom et al., 2003; Gruijters \& Fleuren, 2018). For instance, attitudes are often measured reflectively using 
semantic differentials (Ajzen, 2001; Fishbein \& Ajzen, 2010), such as rating something as "good versus bad", but one could also create an index of an attitude based on specific beliefs people might hold (Fishbein \& Ajzen, 2010). Alternatively, one may also model attitudes as a network of causally interacting evaluations and beliefs (Dalege et al., 2016). Ultimately, the chosen approach depends on the specific indicators that are used, which in turn is primarily constrained by substantive theory. Although networks provide an important addition to the modelling of constructs, we omit network models from the discussion that follows to avoid obfuscating the purpose of this paper.

To formulate that purpose: Authors have long since noted that estimating reliability within a single test administration - using the consistency of responses to different measurements - requires a reflective measurement model (Bollen, 1984; Bollen \& Lennox, 1991; Borsboom, 2006; Edwards, 2011; Falk \& Savalei, 2011; Fleuren et al., 2018; Wilcox et al., 2008). We have noted, however, a pervasive and ongoing tendency to estimate internal consistency for any multiple indicator instrument - regardless of the measurement model. This observation coheres poorly with the notion that internal consistency is only sensible to assess the reliability of reflective models. Whether a reflective measurement model is involved is an essential but often neglected assumption underlying the internal consistency approach. But how can one easily establish whether an instrument has a reflective measurement model? And what are alternatives to assessing reliability when an instrument is not reflective?

This paper aims to address these questions by first reviewing a modified version of Jarvis et al.' (2003) checklist for recognizing reflective measurement (see also Fleuren et al., 2018; MacKenzie et al., 2005). The checklist provides researchers, reviewers, and editors with a tool to determine whether estimates of internal consistency can be meaningfully reported. Secondly, we sketch alternatives to internal consistency estimates to evaluate repeatability of formative measurement. Before describing the checklist and various alternative approaches to reliability assessment of formative measures, we first discuss in more depth the assumption of reflective measurement and its connection to internal consistency. 


\section{Reliability estimation within a single test}

Reliability pertains to the repeatability or stability of a test result (Crocker \& Algina, 1986; Guttman, 1945; Nunnally \& Bernstein, 1994). Analysis of internal consistency (using data from a single test administration) was developed to overcome the practical hurdles involved in examining test-retest reliability (Guttman, 1945). Classical test theory defines an observed score $X$ as a composition of true score $T$ plus error $E$, that is $X=T+E$ (Crocker \& Algina, 1986; Nunnally \& Bernstein, 1994). The observed score variance is accordingly defined as $\operatorname{var}(\mathrm{X})=\operatorname{var}(\mathrm{T})+\operatorname{var}(\mathrm{E})$. Reliability - the extent to which observed score variance overlaps with true score variance $(\rho \mathrm{XT})$ - then, refers to the ratio between true score variance $(\mathrm{T})$ versus observed score variance $(\mathrm{X})$. That is,

$$
\rho \mathrm{XT}=\frac{\operatorname{var}(T)}{\operatorname{var}(T)+\operatorname{var}(E)}
$$

where the observed score variance $X$ equals the sum of true score $(T)$ plus error variance $(E)$.

Typically, several indicators $X$ for true score $T$ are included to achieve a better approximation of $T$, with the assumption that the error $E$ is averaged out so that $X$ will asymptotically approximate $T$. Assuming that each indicator $X$ captures the $T+E$ for the same construct, the indicators are essentially repeated measurements of the same true score. Based on this reasoning — and a set of additional assumptions - the internal consistency of a set indicators approximates an estimate of measurement error at a given test occasion.

The classical definition can be reshaped in terms of a latent variable model as the proportion of variance in composite score $X$ that is explained by a single latent variable (Graham, 2006). Figure 1 depicts structural equation models of three classic measurement models underlying reliability estimation. Reliability of a psychological test with $k=3$ indicators is the proportion of variance in composite $X$ (where $X=X_{1}+X_{2}+X_{3}$ ) explained by latent variable $T$ (to mirror the classical terminology). The latent variable $\mathrm{T}$ can be estimated using distinctive measurement models imposing different constraints. Depicted in Figure 1 are the parallel, (essentially) tau-equivalent, and congeneric models. Panel A illustrates a parallel model, which assumes that indicators have equal factor loadings, equal intercepts, and equal error variances (Cho, 2016; Graham, 2006). The Spearman-Brown formula 
using the split-half correlation, or standardized alpha, can be used to estimate reliability of a composite that is composed of $k$ parallel measures (e.g., Cho, 2016; Falk \& Savalei, 2011).

The historical successor to the parallel model — the (essentially) tau-equivalent model—is depicted in Panel B. By contrast to the parallel model, the tau-equivalent model does not assume that the error variance of each measurement is equal (Cho, 2016; Cortina, 1993; Falk \& Savalei, 2011; Graham, 2006). However, the (essentially) tau-equivalent model also assumes however that each indicator measures the latent variable with equal precision (i.e., equal factor loadings). The difference between tau-equivalent and essentially tau-equivalent pertains to whether the item intercepts are allowed to vary or are assumed equal (see Figure 1, Panel B) — though the difference does not affect estimates of internal consistency. Coefficient alpha provides an estimate of the lower bound reliability in case multiple measurements are (essentially) tau-equivalent (Cho, 2016; Cortina, 1993; Graham, 2006; Peters, 2014). Note that when the factor loadings of the indicators are unequal, then coefficient alpha does not equal the reliability of the composite (Dunn et al., 2014; Hayes \& Coutts, 2020; Peters, 2014; Revelle \& Zinbarg, 2009; Sijtsma, 2009). A third measurement model—-the congeneric modelrelaxes both the equality assumptions involving the factor loadings and error variances. This model is depicted in Panel $\mathrm{C}$ and underlies estimates of internal consistency such as the omega coefficient (Dunn et al., 2014; Hayes \& Coutts, 2020; Peters, 2014; Revelle \& Zinbarg, 2009). 
Figure 1. Measurement models underlying common methods of estimating reliability (see Graham, 2006). $T$ is estimated in a latent variable model imposing different constraints in the respective panels. $X$ is a composite (e.g., sum score) of indicators $X_{\text {i. }}$. The figure uses (unconventional) equality signs between the paths to indicate equality assumptions. Note that each indicator of the composite score $X$ has equal weight (e.g., represented by a sumscore).

\section{A. Parallel}

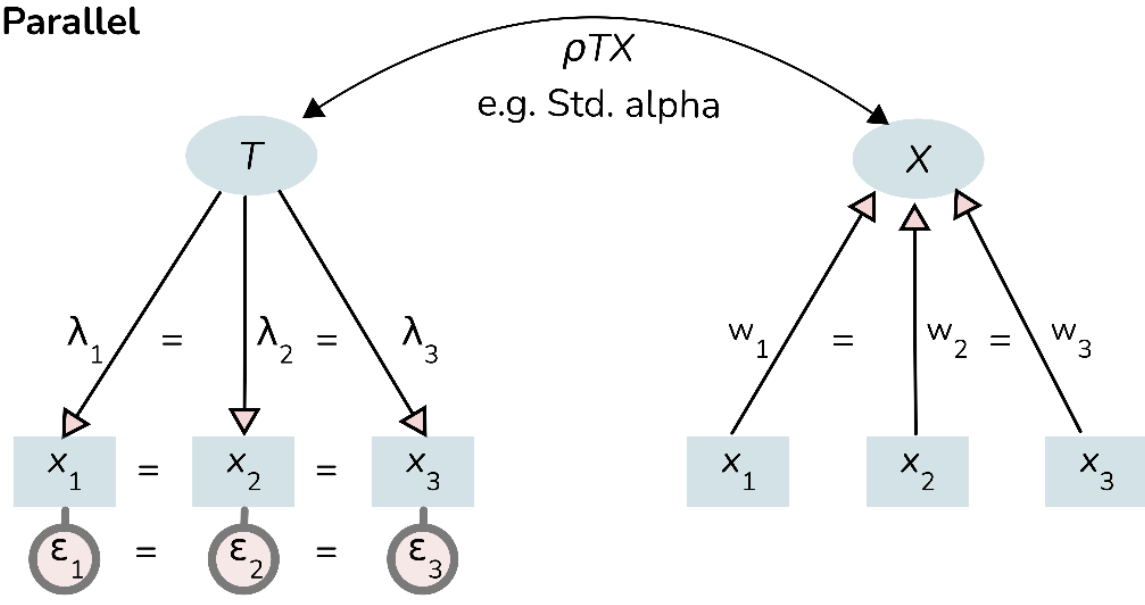

B. (Essentially) Tau-equivalent

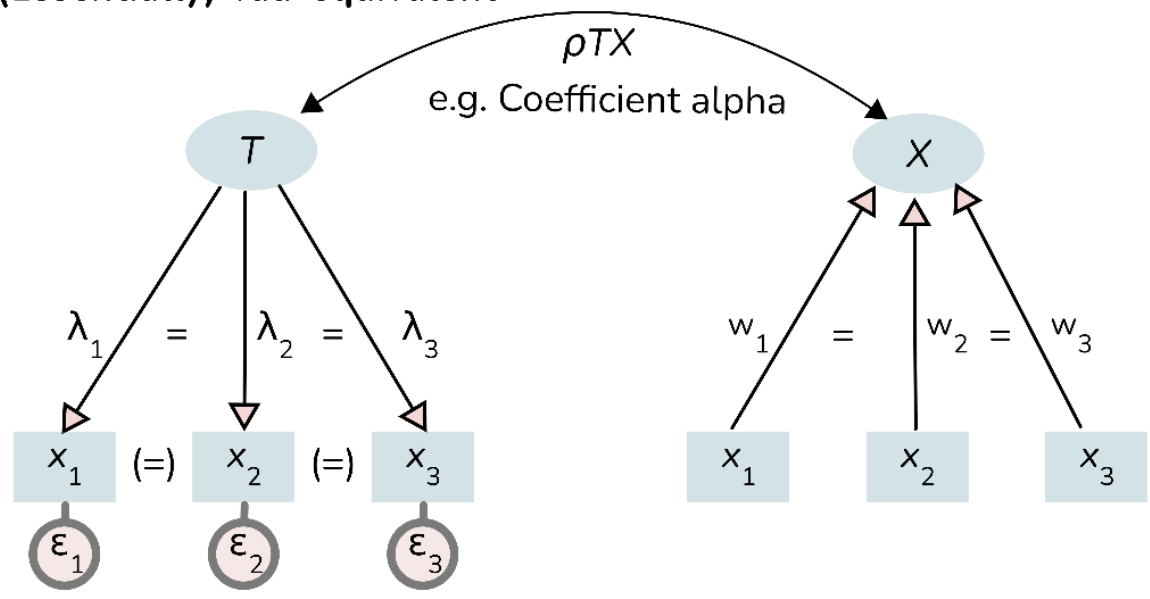

C. Congeneric

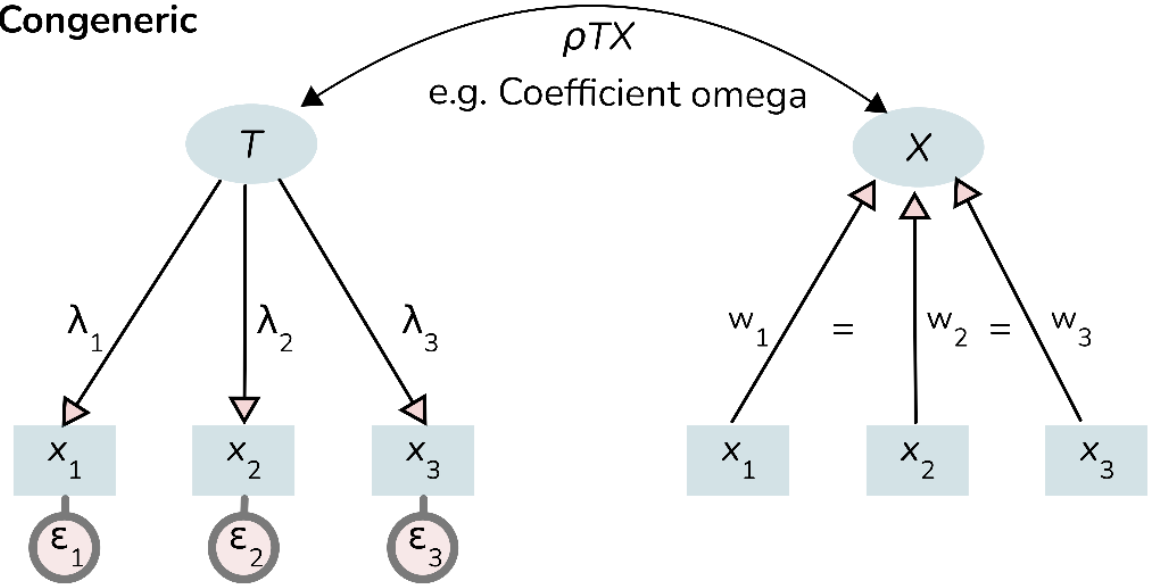




\section{Internal consistency and reflective measurement}

Despite the differences between the parallel, tau-equivalent, and congeneric measurement models underlying internal consistency indices, they share one important assumption—indicators need to be reflective of one latent variable underlying the shared variance (Bollen \& Lennox, 1991; Edwards, 2011; Edwards \& Bagozzi, 2000; Falk \& Savalei, 2011; Fleuren et al., 2018; Jarvis et al., 2003). Figure 1 renders the assumption of reflective measurement is graphically salient by the direction of the arrows between the indicators and the latent variable $T$, which flow downwards from the latent variable to its indicators. Latent variables are identified by examining whether the latent variable renders the indicators locally independent (Bollen, 2002; Borsboom et al., 2003; Gruijters, 2019; Van Bork et al., 2017). Local independence implies that the correlations between indicators disappear when the model partials out (holds constant) the effect of a latent variable. Latent variables - and their ability to generate local independence — come with ontological commitments as they are best understood as common causes of the item covariances (Borsboom et al., 2003, 2004). For instance, a latent 'attitude' variable can be understood as causing the covariance on indicators, such as the degree to which individuals deem something important, worthwhile, or desirable (see Table 1).

In formative models, the causal paths between indicators and construct are reversed compared to reflective measures (Bollen \& Bauldry, 2011; Edwards \& Bagozzi, 2000; Fleuren et al., 2018; Jarvis et al., 2003) ${ }^{1}$. Constructs such as job performance (e.g., Ramos-Villagrasa et al., 2019) or job satisfaction (Spector, 1985) — the latter of which will in this paper be used as a running example — are typically measured by inquiring about distinctive aspects of the job. Such aspects, for example workers' satisfaction with pay, communications, supervision, co-workers and so forth (Spector, 1985), are best seen as defining features of overall satisfaction with the job (see also MacKenzie et al., 2005). Even though it is possible to measure an individual's general level of job satisfaction with reflective items (e.g., I generally feel satisfied in my daily work), instruments often rely on indicators that compose rather than reflect job satisfaction (see Table 1). Composites of formative items are perhaps best interpreted as producing instrumental summary variables or practically useful indices, rather than as providing measurements of latent variables (see Howell et al., 2007; Rhemtulla et al., 2015). 
The measurement literature connects the idea of internal consistency exclusively to the reflective model (Bollen \& Lennox, 1991; Edwards, 2011; Edwards \& Bagozzi, 2000; Falk \& Savalei, 2011; Fleuren et al., 2018; Jarvis et al., 2003; Wilcox et al., 2008). The gist of the arguments to do so is that estimating measurement error within a single test assumes that each indicator provides either a direct or a conceptual replication of the others. This follows from the unidimensionality assumption. Indicators in a (sub)test are required to reflect a single latent variable — or, in terms of classical test theory, estimate a single true score (T). Consequently, indicators in reflective tests must either be identical or conceptual replications, since being caused by the same latent variable implies such conceptual overlap. Because of sharing a common cause, we can expect that in an ideal world (free of measurement error and other disturbances) such indicators would correlate perfectly with one another. Accordingly, when indicators are reflective they are-at least principally—interchangeable and redundant. Therefore, removing or adding reflective indicators should not substantially alter the meaning of the factor or composite scores.

These notions underlie the rationale for estimating internal consistency, as such estimates use the correlations between indicators to estimate the amount of measurement error. Accordingly, the error-term assigned to each indicator is estimated based on a regression of the factor variance to the indicators. Conversely, factor analytic notions such as unidimensionality, local independence, and that indicators directly or conceptually overlap are often not (and need not be) fulfilled by formative models. Notably, internal consistency is not required, desired or expected for instruments containing formative indicators.

\section{Checklist: is it meaningful to estimate internal consistency?}

To assess whether internal consistency estimates are sensible to calculate, researchers, reviewers or editors, first need to determine whether indicators form a reflective measurement model. We recommend that researchers use a (modified version of) checklist (orginally developed by Jarvis et al. 2003) which contains seven checks of reflective measurement (see Table 2). It is essential to ask these questions for each item separately, as it may be possible that an instrument contains a combination of both reflective and formative indicators. The checklist is itself supposed to show high internal consistency because the indicators are conceptually closely overlapping - all indicators 
pertain, one way or another, to the direction of causation between construct and indicators. Reflective tests should in principle obtain a score of seven points; where each 'yes answer' is awarded a single point. This is not a clear dichotomy, however. That is, one or two criteria might be scored as 0 ("no"), because formative measures can (but need not) have for instance correlated indicators. The redundancy of the indicators in the checklist should ensure a proper assessment of whether a scale is likely reflective. We discuss the modified Jarvis et al (2003) checklist in turn with two examples from the literature-items measuring attitudes, and an items designed to assess job satisfaction (see Table $1)$. 


\section{ASSESSING THE RELIABILITY OF FORMATIVE INSTRUMENTS}

Table 1. Example indicators used in an instrument measuring 'attitude towards colleague contact' and 'job satisfaction'.

\begin{tabular}{lll}
\hline Item \# & Attitude & Job satisfaction \\
& semantic differential scale & My supervisor shows too little interest in the feelings of subordinates. \\
\hline 1 & I think ample contact with colleagues is... & \\
& $(1=$ bad; $5=$ good $)$ & Communications seem good within this organization \\
& I think regular interactions with colleagues is... & \\
& $(1=$ not worthwhile; $5=$ worthwhile $)$ & My supervisor is unfair to me \\
& I think spending time with colleagues is... & \\
& $(1=$ unpleasant; $5=$ pleasant $)$ & The goals of this organization are not clear to me.
\end{tabular}

Note. Attitude items are constructed according to Fishbein \& Ajzen (2010). The job satisfaction items are selected from the Job Satisfaction Survey (Spector, 1985). 


\section{Construct - indicator relationship}

The first three question inquire about the relationship between the measured construct and the items included in the instrument, albeit in distinctive phrasings. To assess how items relate to a construct requires an explicit theory of the construct at hand, including a precise definition. An essential feature of reflective indicators, then, is that the content of items can be considered a manifestation of the construct of interest - in the attitude example, that attaching importance to something is a concrete manifestation of a positive attitude, and vice versa. Formative items are better seen as defining features of a construct, they combine different sources of information into a composite score. Hence, the first question inquires whether each and every item in the instrument can be considered a manifestation of the construct.

\section{Causal direction}

The second question (causal direction) explicates the nature of the relationship between construct and indicators further in terms of causality. As discussed in a previous section, several authors have conceptualized reflective measurement in causal terms (e.g., Bollen \& Lennox, 1991; Borsboom et al., 2003; Edwards \& Bagozzi, 2000), in which variance in an indicator (e.g., how important is action $\mathrm{x}$ ) is conceptualized as being caused by variance in the construct, whereas the direction of causality is reversed in formative indicators. This question thus asks whether it is reasonable to point the causal arrow away from the latent variable towards the indicators, so that it is sensible to view the construct as a common cause of the item variance. One way to assess is this by specifically considering a traditional condition for causation, temporal precedence (e.g., Campbell, 1957; Shadish et al., 2002). In the case of job satisfaction, this would imply asking which came first, variations in satisfaction with the job and only later variation in contentment with the salary, or vice versa?

\section{Counterfactual dependence}

This third question elaborates and further assists with determining the direction of causality between construct and indicators, but does so using the counterfactual approach to detecting causation (Pearl \& Mackenzie, 2018; Woodward, 2003). According to this approach, a causal relationship can be detected by considering what would happen under a counterfactual circumstance-would different 
scores on the construct imply different scores on the indicator, or vice versa? One way to assess this is by conducting a thought-experiment: what if one would push upwards (by intervention) the values on the construct towards some level (e.g., push upwards people's attitude), would this result in different scores on the indicator? Or would an intervention on the indicator result in different values for the construct? Of course, although a thought-experiment may help to detect the possibility of a causal association in the required direction — such a possibility represents a necessary but not sufficient condition for a causal association (Woodward, 2003).

\section{Conceptual homogeneity}

The fourth checklist question enquires about implications of reflective measurement on the instrument level. The item asks about conceptual overlap between the indicators, some degree of which is expected for a set of reflective indicators but not for formative ones. Consider that each indicator is supposed to be sensitive to variance in the same latent variable. Sharing such a common cause (i.e., responsiveness to the same latent variable) implies some level conceptual similarity—or, homogeneity. For instance, the semantic differential, "unimportant-important" versus "undesirabledesirable" are overlapping in one way because the response model assumes that such ratings are causally influenced by individuals' overall disposition to respond in particular way to an object or action. Formative instruments often include defining features or causes of a construct, some of which may be conceptually related whereas others are not (e.g., payment satisfaction versus perceived communication quality). Thus, conceptual homogeneity is possible but not necessarily present in formative instruments.

Note that there is an intricate relationship between conceptual homogeneity and statistical expectations such as unidimensionality and internal consistency. Cortina (1993) rightly cautions that internal consistency is a necessary but not a sufficient condition for homogeneity. Thus, while conceptually homogenous instruments are expected to exhibit high degrees of internal consistency, internal consistency does not imply that a measure therefore contains conceptually homogenous items. The same points apply to the distinction between reflective and formative indicators. Conceptual homogeneity represents a necessary but insufficient condition of reflective measures-since it is principally possible to design an instrument with homogenous formative indicators. As conceptual 
homogeneity is related to the expectation of unidimensionality (Cortina, 1993), this further implies that unidimensionality is also not sufficient to conclude that an instrument uses reflective indicators. In short, conceptual homogeneity, internal consistency, and unidimensionality are all necessary, but not sufficient conditions for reflective measurement.

\section{Correlations between indicators}

If $\mathrm{k}$ indicators are causal manifestations of the same construct, such a circumstance further implies that indicators correlate substantially (Bollen, 1984; Bollen \& Lennox, 1991) - the fifth item in the checklist. This also follows directly from the discussed requirement of homogeneity, a statistical symptom of which is to observe substantial correlation between the items. Conversely, this is not necessarily the case for formative indicators - formative indicators may or may not correlate to some extent (Bollen \& Lennox, 1991; Edwards, 2011; Jarvis et al., 2003; Wilcox et al., 2008).

Besides that substantial correlation is expected between reflective indicators, another implication involving the correlations between reflective indicators underlies the rationale of the vanishing tetrad test (VTT), or confirmatory tetrad analysis (Bollen \& Ting, 1993, 2000). A tetrad is

estimated by subtracting the product of two item population covariances $\left({ }_{i j} \sigma_{k l}\right)$ from the product of another pair of items. This is done for each non-redundant tetrad given the $k$ assumed reflective indicators. For instance, for $k=4$ reflective indicators there are six covariances between indicators.

These can be arranged in three tetrads $\left({ }^{\tau_{i j k l}}\right)$ :

$$
\begin{aligned}
& \tau_{1234}=\sigma_{12} \sigma_{34}-\sigma_{13} \sigma_{24} \\
& \tau_{1342}=\sigma_{13} \sigma_{42}-\sigma_{14} \sigma_{32} \\
& \tau_{1423}=\sigma_{14} \sigma_{23}-\sigma_{12} \sigma_{43}
\end{aligned}
$$

where ${ }^{\sigma_{12}}$ denotes the population covariance between two indicators. Bollen \& Ting $(1993,2000)$ have argued that in case of reflective — but not for formative-measurement the population tetrads such as $\tau_{1234}$ are expected to be zero. Measurement models can be tested with CTA for this implication using various statistical approaches (Bauldry \& Bollen, 2016; Bollen \& Ting, 2000). CTA 
provides supportive evidence of reflective measurement - it provides a means to empirically test the fit of the model implied by reflective measurement (for a practical illustration; Bollen et al., 2009).

\section{Conceptual exchangeability and interchangeability}

The statistical and conceptual overlap between reflective indicators also implies some level of redundancy: removing an indicator does not imply a substantive change in the measurement result (Fleuren et al., 2018; Jarvis et al., 2003). Conversely, removing a conceptually and statistically independent formative indicator may change the interpretation of the construct. For instance, whether one uses four or five conceptually overlapping — and highly correlating —indicators of attitude (worthwhile, important, good, desirable) may not substantively change the measurement outcome (individuals' relative position on the composite for instance). But removing a specific formative indicator (e.g., satisfaction with pay) from a conceptually and perhaps statistically independent set of indicators could change the meaning of the resulting composite. One way to assess the interchangeability of items is to generate random subsets of the $\mathrm{k}$ indicators: would any randomly generated subset of $\mathrm{k}$ items (for instance by a random split-half) be equivalent to the original scale in terms of construct interpretation and strength of association with other variables? The sixth indicator of the checklist covers this issue of inter- and exchangeability.

\section{Criterion relationships}

The similarity between indicators further suggests something about the relationships of the individual items with a criterion variable. Specifically, suppose a robust relationship has been established between the attitude towards colleagues composite scores and various outcomes (e.g., well-being or performance). Because of their conceptual overlap, in sharing a common cause, one expects that regressing individual indicators on external criteria would (at least by expectation) result in a similar pattern of associations also for each indicator separately (keeping measurement error constant). Another way to interpret this final question is to ask whether each and every indicator in the instrument could principally be used as an equivalent single-measure predictor of the construct of interest. 


\section{Checklist implications}

What course of action should researchers take when the checklist indicates that one or more indicators are non-reflective? We envision two distinctive uses of the checklist—a priori to guide development and selection of instruments, and a posteriori, to evaluate the feasibility of the reporting of internal consistency estimates. As a general principle, our presupposition is that reflective measurement comes with various advantages to researchers in psychology, as the epistemological value of reflectively measured constructs is more transparent (Edwards, 2011; Hardin, 2017; Hardin \& Marcoulides, 2011; Howell et al., 2007). Put more concretely, suppose one creates a composite score of the job satisfaction scale and finds that the composite predicts the employees' overall well-being. The formative nature of the items invites examining the relevance of the composite indicators separately. Given that, being conceptually and perhaps statistically independent, formative indicators may have different causes, consequences, and interactions. By implication, we recommend caution in the use of composite scores of formative indicators and to additionally consider indicator-level analyses. Overall, we recommend that—unless an explicit rationale or theory for using a composite of particular formative indicators is at hand - reflective measurement of the various facets is best first attempted as to avoid the various complications encountered in formative measurement (Edwards, 2011; Fleuren et al., 2018; Jarvis et al., 2003). This may either be achieved by a priori instrument selection, or by respecification of a formative model into a reflective model (Edwards, 2011; Jarvis et al., 2003). 
ASSESSING THE RELIABILITY OF FORMATIVE INSTRUMENTS

Tabel 2. Application of a modified version of Jarvis et al's (2003) checklist to a job satisfaction and attitude measure

\begin{tabular}{ll}
\hline Question & Example (attitude versus job satisfaction; see Table 1) \\
\hline Construct - indicator relationship & $\checkmark$ Reponses to semantic differentials (e.g., "I think ample contact with colleagues is important / \\
Are the indicators manifestations of the construct? (Yes / No) & unimportant”) could be considered manifestations of latent 'attitude' variable.
\end{tabular}

$\times$ Satisfaction with the communication within an organization can be one of the building blocks of overall job satisfaction, but it is not a direct manifestation of it.

\section{Causal direction}

Is construct variance causally responsible for variance in the indicators? (Yes / No)

\section{Counterfactual dependence}

If one would perform an intervention to change values on the construct, would this lead to a change on the value(s) of the indicator(s)? (Yes / No)

\section{Conceptual homogeneity}

Are the indicators conceptually closely related to each other in terms content domain? (Yes / No)

\section{Correlations between items}

Do increasingly higher values on the indicator necessarily imply a higher probability of higher values on the other indicators? (Yes / No) $\checkmark$ Differences between individuals' relative value on latent attitude variable can be viewed as causally generating variance on specific semantic differentials.

× Scores on a latent 'job satisfaction' variable are not likely a direct cause of satisfaction with the communications within a company.

$\checkmark$ If one intervenes on the attitude of a group of individuals, we could reasonably expect these individuals to show different responses to semantic differentials measuring that attitude.

x If one intervenes to improve levels of job satisfaction, they will not necessarily respond different to questions covering the quality of communications, supervision, or satisfaction with pay.

$\checkmark$ Semantic differentials (good/bad, important/unimportant, desirable/undesirable, etc.), with regard to the same objects or activity, are concepts that overlap closely in meaning.

× Job satisfaction indicators, such as 'communications quality', and 'satisfaction with pay' have different extension and cover different conceptual domains.

$\checkmark$ For a given set of k semantic differentials, statistical dependence between the indicators is expected. Individuals with scores on one indicator are expected to correlate with higher scores on other items.

x For the Job satisfaction scale, scores on questions about the quality of communications within a company need not be correlated to how satisfied these individuals are with their salary. It is not necessarily expected that such items correlate. 


\section{Conceptual exchangeability / interchangeability}

Are the indicators a random selection of conceivable indicators of the construct? That is, are they roughly interchangeable and exchangeable? (Yes / No)

\section{Criterion relationships}

Is it expected that indicators exhibit empirical convergence and divergence with the same criterion variables? (Yes / No) $\checkmark$ The attitude indicators are not collectively exhaustive of the construct, but can be seen as a random set of indicators drawn from a larger pool. Deletion or replacement of a single item is inconsequential to the conceptual interpretation of the construct. By implication, the indicators can be combined into subsets at random while providing equivalent measurements, albeit possibly with varying reliability.

$\times$ The Job satisfaction indicators collectively define the construct - as they ask about different aspects related job satisfaction. Dropping particular indicators may affect the conceptual interpretation of the test, because particular conceptual domains may not be covered anymore after removal. The interpretation of what is measured by the factor scores could change as a consequence.

$\checkmark$ If one uses k semantic differentials (good/bad, important/unimportant, desirable/undesirable, etc.) to measure attitude toward the same object or activity, each indicator is expected to be associated — by a similar degree — with a criterion.

x If one measures job satisfaction with conceptually, and perhaps statistically, independent indicators (e.g., communications quality, satisfaction with pay) there is no implication that such indicators relate to a similar degree to criterion variables. 


\section{ASSESSING THE RELIABILITY OF FORMATIVE INSTRUMENTS}

\section{The alternative of test-retest reliability}

We have argued that the internal consistency approach is unfounded when formative indicators are in play. Under various circumstances however, the use of formative instruments may be warranted, and perhaps unavoidable. In such instances, there is value to examining the reliability of the formative instrument with alternative methods. One suitable alternative which does not hinge on the assumption of reflective measurement is test-retest reliability (Crocker \& Algina, 1986; Guttman, 1945; Nunnally \& Bernstein, 1994; Schmidt et al., 2003; Weir, 2005).

The within versus between tests approaches offer distinct ways of looking at measurement error. There are different sources of measurement error, including random response error (e.g., attention lapses, fatigue), specific-factor errors (e.g., participants' idiosyncrasies in responding to specific items) and transient error (e.g., occasion dependent state of mind of the participant), among others (Schmidt et al., 2003; Schmidt \& Hunter, 1999). Transient error (e.g., Chmielewski \& Watson, 2009) refers to variation in responses caused by 'random variations in respondents' psychological states across time (Schmidt et al., 2003, p. 206). In statistical terms, transient error refers to the interaction between person and test occasion. The test-retest approach captures both the combined measurement error due to random response error and transient error, but not specific-factor error.

Conversely, internal consistency estimates are sensitive to a combination of random response error and specific-factor error, but cannot capture transient error. Because within and between test reliability estimates capture different sources of error, an optimal approach is to utilize a combination of both (see Cronbach, 1951; Schmidt et al., 2003). Such a combined approach is however rare in the literature-reliability estimation typically proceeds using the internal consistency approach. In the present context of formative instruments, the internal consistency approach (and therefore neither the combined approach) is for reasons discussed in the previous sections not feasible. Using test-retest reliability estimation is therefore an acceptable solution in the case of formative instruments.

\section{Assessing the consistency of a formative instrument over time}

The Pearson's correlation between a test and retest of a formative instrument indicates whether ordinal consistency exists in the composite score over time (Crocker \& Algina, 1986; Nunnally \& Bernstein, 1994). A more flexible approach to estimating test-retest reliability is offered by Intraclass 
Correlation Coefficients (ICC; Bartko, 1976; Cronbach \& Shavelson, 2004; McGraw \& Wong, 1996; Shieh, 2016; Shrout \& Fleiss, 1979; Weir, 2005). Compared to Pearson's r, an important of advantage of the ICC is that it allows analyzing more than two test occasions. Interactions between items and person, or test occasions and persons, can also be evaluated using ICC. ${ }^{2}$ Additionally, ICC can also be adjusted for systematic sources of error. An ICC estimates the reliable proportion of variance (attributable to 'real' differences between participants). The basic population ICC for reliability can be described as:

$$
I C C=\frac{\sigma^{\text {persons }}}{\sigma^{\text {persons }}+\sigma^{\text {error }}}
$$

Theoretically the ICC ranges between $0-1$ with random measurement error defined as the complement (1-ICC). Note that the basic formulation of an ICC (Equation 2) for reliability mirrors the definition of classical test theory (Equation 1) — though true score variance is replaced by a conceptually similar between-persons variance estimate (Brennan, 2005). For our present purposes, we refrain from a more detailed review of the various ICC forms - excellent and detailed discussion of the various ICC is found in Shrout \& Fleiss (1979) and McGraw \& Wong (1996).

If one has test-retest data there are (at least) three variance components that can be estimated using ANOVA. One can distinguish between variance due to differences between persons (i.e., row variance; MSR), differences between test occasions (i.e., column variance; MSC) and error variance (MSE). An ICC for consistency (C,1) can be used as an estimate of the coefficient of stability (e.g., Weir, 2005) ${ }^{3}$ :

$$
I C C(C, 1)=\frac{M S R-M S E}{M S R+(k-1) M S E}
$$

which is identical to ICC $(3,1)$ in terms of Shrout and Fleiss (1979). When applied to two-wave testretest data, the estimated ICC for consistency equals the Pearson's r estimate.

Assessing agreement with the intraclass correlation

Under the hypothetical scenario of an $\operatorname{ICC}(C, 1)=1$, or Pearson's $r=1$, one may be tempted to conclude that participants' scores are perfectly reproduced over time. However, this is only so when 
the test occasions (e.g., T1 and T2) have equal mean values (e.g., Cohen's d = 0). Consider the two measurements depicted in Figure 1. Both panels A and B are perfectly correlated $(r=1)$, indicating the absence of random measurement error. However, whereas both panels exhibit perfect reliability, the results shown in panel B show systematic error (as estimated by the mean difference). For instance, in the example of job satisfaction the construct may have changed systematically in the research population - which could conceivably occur when participants receive an increase of salary between measurements (see also Barber et al., 2013). Put differently, coefficients of stability (ICC(C,1) or Pearson's r) estimate what Mcgraw \& Wong (1996) describe as a linearity index - the extent to which the composite scores on one measurement can linearly be related to scores on a second measurement. Such estimates, however, cannot detect the presence of systematic error over time.

Figure 1. Scatterplot of two simulated test-retest datasets $(n=200)$. In both panels A and B estimated measurement error is zero $(r=1)$. Systematic error (mean difference) in Panel A is absent (Cohen's $d=0)$, whereas the data in Panel B contains bias (Cohen's $d=0.67)$.

A.

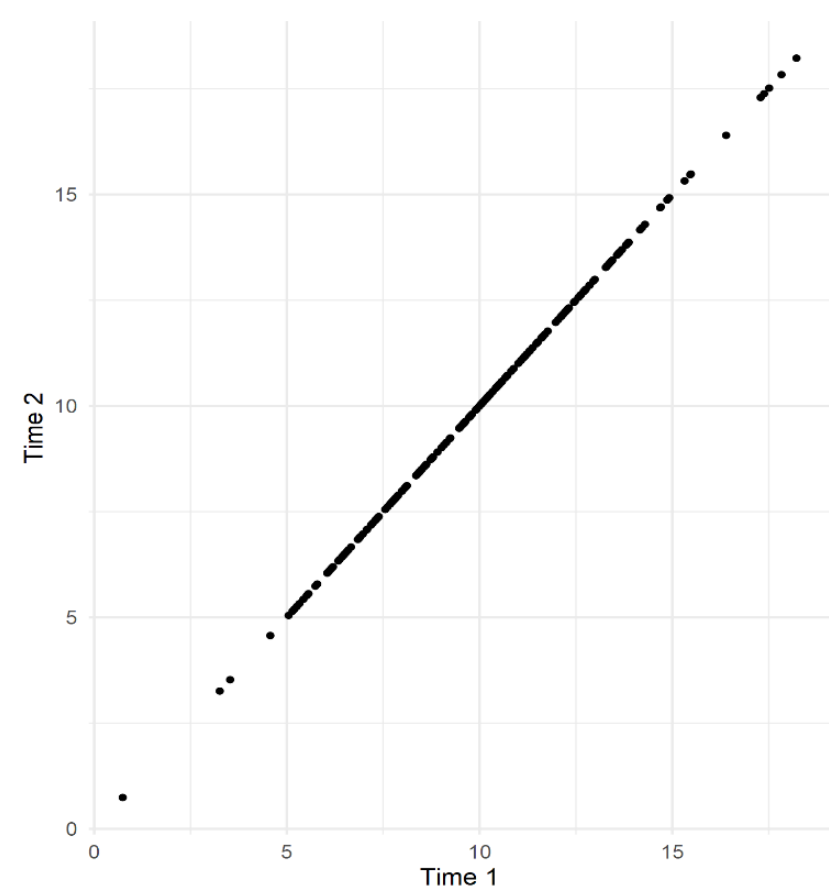

B.

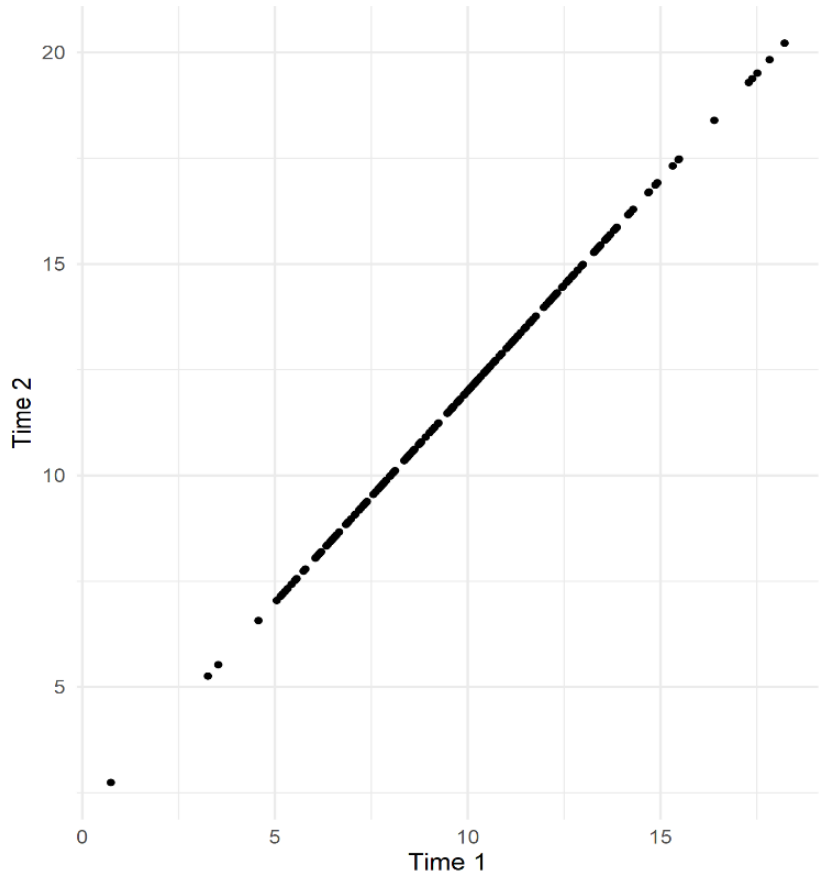

To detect systematic error, an ICC for absolute agreement can additionally be estimated

(McGraw \& Wong, 1996; Shrout \& Fleiss, 1979). Considering the trials a fixed variable, then ICC(A,1) estimates absolute agreement of the composite scores between the trials: 


$$
\operatorname{ICC}(A, 1)=\frac{M S R-M S E}{M S R+(k-1) M S E+\frac{k}{n}(M S C-M S E)}
$$

which is equivalent to ICC(2,1) in Shrout \& Fleiss' (1974) nomenclature. To reiterate the difference between $\operatorname{ICC}(\mathrm{C}, 1)$ and $\operatorname{ICC}(\mathrm{A}, 1)$ : the latter includes column variance (mean shifts over time) in the denominator, which will—in the presence of systematic error—lower the reliability estimate. By comparing the estimated $\operatorname{ICC}(\mathrm{A}, 1)$ with the $\operatorname{ICC}(\mathrm{C}, 1)$ it is possible to assess the extent of systematic error - since both ICC estimates will be equal when the mean shift over time is zero. In Figure 2, the results of two simulated test-retest studies are depicted. Panel A depicts two matched composite scores for two measurement occasions which are unbiased - the mean for measurement B equals that of measurement A. In panel B shows another test-retest data which exhibits systematic error-a substantial mean difference of Cohen's $d=0.64$ in this case. Even though the two scatterplots show equivalent Pearson's and ICC $(\mathrm{C}, 1)$ based consistency, the two plots differ substantively in terms of absolute agreement between the two measurements (see Table 3).

Figure 2. Two simulated datasets with correlated repeated measurements of a composite score. $\mathrm{N}=200$ and Pearson's $\mathrm{r}=.84$ in both panels.

A

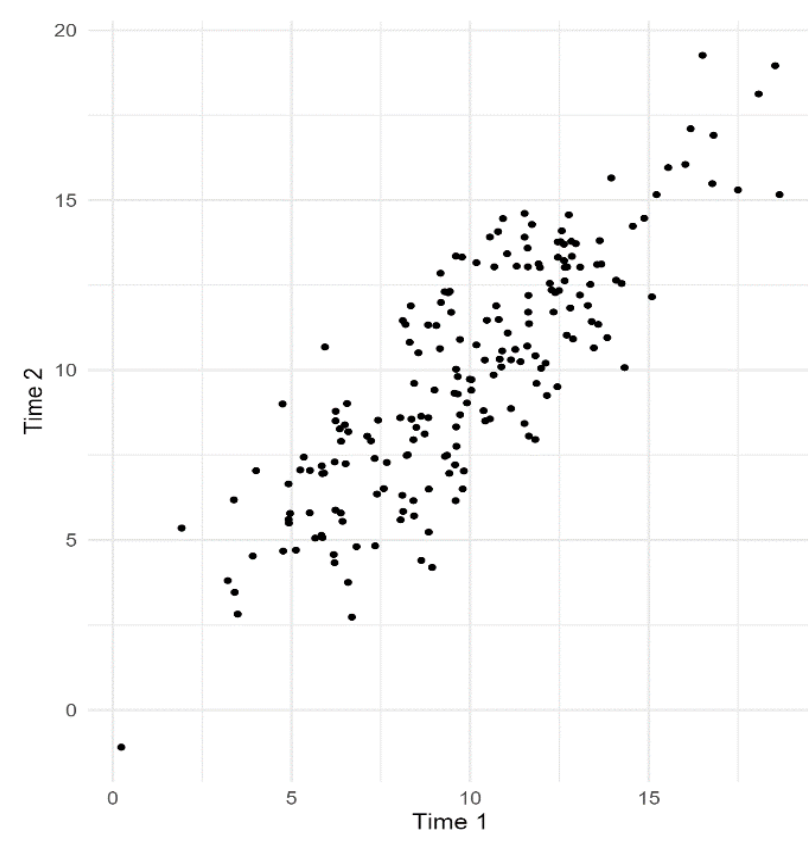

B

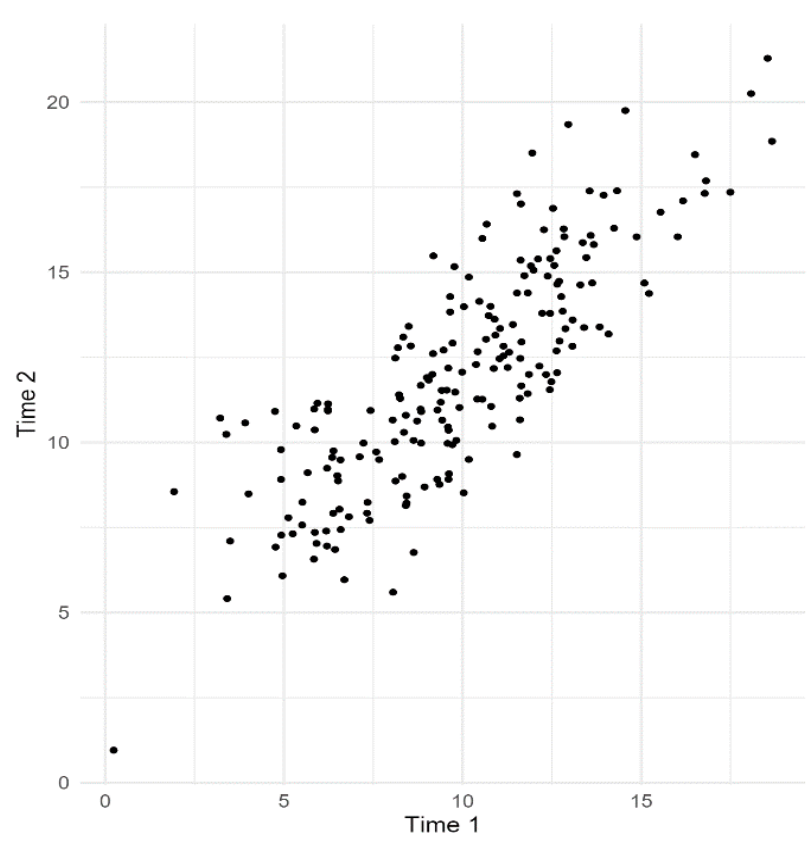


Table 3. Data and correlation estimates corresponding to Figure 2.

\begin{tabular}{|c|c|c|c|}
\hline Measurement 1A & Measurement 2A & Measurement 1B & Measurement 2B \\
\hline $\mathrm{M}=9.90$ & $\mathrm{M}=9.92$ & $\mathrm{M}=9.90$ & $\mathrm{M}=12$ \\
\hline $\mathrm{SD}=3.3$ & $\mathrm{SD}=3.46$ & $\mathrm{SD}=3.3$ & $\mathrm{SD}=3.3$ \\
\hline $\mathrm{n}=200$ & $\mathrm{n}=200$ & $\mathrm{n}=200$ & $\mathrm{n}=200$ \\
\hline Correlation & Calculation & Correlation c & interpretation \\
\hline \multirow[t]{2}{*}{ Pearson's $r=.84$} & $\operatorname{cov}(x, y)$ & Pearson's $\mathrm{r}=.83$ & Single measure \\
\hline & $\sigma_{x} \sigma_{y}$ & & Consistency \\
\hline \multirow[t]{2}{*}{$\operatorname{ICC}(C, 1)=.84$} & $M S_{R}-M S_{E}$ & $\operatorname{ICC}(C, 1)=.83$ & Single measure \\
\hline & $M S_{R}+(k-1) M S_{E}$ & & Consistency \\
\hline \multirow[t]{2}{*}{$\operatorname{ICC}(\mathrm{A}, 1)=.84$} & $M S_{R}-M S_{E}$ & $\operatorname{ICC}(\mathrm{A}, 1)=.69$ & Single measure \\
\hline & $M S_{R}+(k-1) M S_{E}+\frac{k}{n}\left(M S_{c}-M S_{E}\right)$ & & Agreement \\
\hline
\end{tabular}

Note. The example calculations are available on the Open Science Framework at https://osf.io/7a95b/?view_only=abc5f0dc54b4476d8890ee9f6f0d2fe4. Estimates and ICC abbreviations are from Mcgraw \& Wong (1996). ICC(C,1)= consistency index for single measures. $\operatorname{ICC}(\mathrm{A}, 1)=$ absolute agreement index for single measures. The ICC are calculated using the "psych" R-package (Revelle, 2020).

\section{Computing the ICC in practice}

One straightforward way to compute the various forms of the ICC is using the "psych" R package (Revelle, 2020), specifically the function "psych::ICC()". This package can be installed with:

$$
\text { install.packages("psych") }
$$

Once installed, the "psych::ICC()" function can be called. For example, assuming the data are stored in a data frame called "data" and the two test administrations are named "scores_t1" and "scores_t2", one would use the following command to obtain the ICC estimates:

psych::ICC(data[, c("scores_t1", "scores_t2")]) 
This will provide six estimates of the ICC (including confidence intervals). In this package, the ICC2 corresponds to what we have labelled here the ICC(A,1), whereas the ICC 3 in "psych" estimates the $\operatorname{ICC}(\mathrm{C}, 1)$.

\section{Practical usage of the test-retest ICC}

An estimate of the ICC — falling between zero and one - provides information on the balance between reliable variance and measurement error variance. For practical purposes, it may be helpful to anchor ICC values in categories of "sufficiently" or "insufficiently" reliable. However, given various objections to benchmarking context-dependent statistical information (e.g., Gruijters \& Peters, 2020), in particular for the different ICC forms (Weir, 2005), we refrain from providing any such cutoffs on when a formative instrument is reliable in an absolute sense. Instead, we note that — similar to internal consistency estimates for reflective measures - a test-retest ICC $(\mathrm{C}, 1) 4$ estimate can be put to practice in several ways. One important implication of random measurement error is its attenuating effect on the observed correlation between the instrument with criterion variables. In classical test theory (see also Baguley, 2009; Bobko et al., 2001), the relationship between reliability and the observed and true correlation is formulated as:

$$
r X_{t} Y_{t}=r X Y \sqrt{r X X r Y Y}
$$

where the attenuated correlation $(r X Y)$ is a function of the true correlation $r X_{t} Y_{t}$ and the respective reliabilities of $X$ and $Y$ (Crocker \& Algina, 1986; Nunnally \& Bernstein, 1994). Assuming both $X$ and $Y$ are formative indices — whose reliability have been estimated with a ICC $(\mathrm{C}, 1)$ - the observed correlation between the formative indices can be represented as:

$$
r X Y=r X_{t} Y_{t}\left(I C C^{2}\right)
$$

A simple rearrangement allows one to disattenuate an observed correlation for unreliability:

$$
r X_{t} Y_{t}=\frac{r X Y}{I C C^{2}}
$$

Another tangible use of an estimated ICC for a formative measure is that its estimate of measurement error can be involved in a priori sample size computations for power or precision. It is has been shown that —under ideal and large N conditions (Loken \& Gelman, 2017)—measurement error decreases the 
size of the correlation and the resulting the power of a statistical test; inflating the required sample size (e.g., Perkins et al., 2000; Phillips \& Jiang, 2016). Estimates of reliability can hence be used in a priori sample size calculations to correct for measurement error adjusting the estimate of the required sample size. Additionally, such estimates of measurement error may also provide useful information towards correcting for attenuation in the context of meta-analysis (e.g., Cumming, 2012).

\section{Concluding remarks}

The distinction between reflective and formative measurement has received ample attention in the measurement literature, but such discussion has mostly been confined to the "niche" measurement and structural equation modelling literatures. For this and other reasons, various implications of the theoretical distinction between reflective and formative measurement have only slowly begun to trickle down towards applied psychometric practice in organizational psychology. This paper attempts to provide a practical resource for researchers, reviewers, and editors to detect reflective measurement — as reflective measurement underlies the rationale for estimating indices of internal consistency. The ICC has been presented as a viable alternative to assess reliability-including of formative measurements— based on a test-retest approach. 


\section{References}

Ajzen, I. (2001). Nature and operation of attitudes. Annual Review of Psychology, 52(1), 27-58. https://doi.org/10.1146/annurev.psych.52.1.27

Baguley, T. (2009). Standardized or simple effect size: What should be reported? British Journal of Psychology, 100(3), 603-617. https://doi.org/10.1348/000712608X377117

Barber, L. K., Barnes, C. M., \& Carlson, K. D. (2013). Random and Systematic Error Effects of Insomnia on Survey Behavior. Organizational Research Methods, 16(4). https://doi.org/10.1177/1094428113493120

Bartko, J. J. (1976). On various intraclass correlation reliability coefficients. Psychological Bulletin, 83(5), 762-765. https://doi.org/10.1037/0033-2909.83.5.762

Bauldry, S., \& Bollen, K. A. (2016). tetrad: A Set of Stata Commands for Confirmatory Tetrad Analysis. Structural Equation Modeling: A Multidisciplinary Journal, 23(6), 921-930. https://doi.org/10.1080/10705511.2016.1202771

Bobko, P., Roth, P. L., \& Bobko, C. (2001). Correcting the Effect Size of d for Range Restriction and Unreliability. Organizational Research Methods, 4(1), 46-61. https://doi.org/10.1177/109442810141003

Bollen, K. A. (1984). Multiple indicators: Internal consistency or no necessary relationship? Quality and Quantity, 18(4). https://doi.org/10.1007/BF00227593

Bollen, K. A. (2002). Latent variables in psychology and the social sciences. Annual Review of Psychology, 53, 605-34. https://doi.org/10.1146/annurev.psych.53.100901.135239

Bollen, K. A., \& Bauldry, S. (2011). Three Cs in measurement models: causal indicators, composite indicators, and covariates. Psychological Methods, 16(3), 265-284. https://doi.org/10.1037/a0024448

Bollen, K. A., \& Lennox, R. (1991). Conventional wisdom on measurement: A structural equation perspective. Psychological Bulletin, 110(2), 305-314. https://doi.org/10.1037/00332909.110.2.305 
Bollen, K. A., Lennox, R. D., \& Dahly, D. L. (2009). Practical application of the vanishing tetrad test for causal indicator measurement models: An example from Health Related Quality of Life. Statistics in Medicine, 28(21), 2724-2724. https://doi.org/10.1002/sim.3662

Bollen, K. A., \& Ting, K.-F. (1993). Confirmatory Tetrad Analysis. Sociological Methodology, 23, 147. https://doi.org/10.2307/271009

Bollen, K. A., \& Ting, K. (2000). A tetrad test for causal indicators. Psychological Methods, 5(1), 322. https://doi.org/10.1037/1082-989X.5.1.3

Borsboom, D. (2006). The attack of the psychometricians. Psychometrika, 71(3), 425-440. https://doi.org/10.1007/s11336-006-1447-6

Borsboom, D., \& Cramer, A. O. J. (2013). Network Analysis: An Integrative Approach to the Structure of Psychopathology. Annual Review of Clinical Psychology, 9(1), 91-121. https://doi.org/doi:10.1146/annurev-clinpsy-050212-185608

Borsboom, D., Cramer, A. O. J., Schmittmann, V. D., Epskamp, S., \& Waldorp, L. J. (2011). The Small World of Psychopathology. PLoS ONE, 6(11), e27407. https://doi.org/10.1371/journal.pone.0027407

Borsboom, D., Mellenbergh, G. J., \& van Heerden, J. (2003). The theoretical status of latent variables. Psychological Review, 110(2), 203-219. https://doi.org/10.1037/0033-295X.110.2.203

Borsboom, D., Mellenbergh, G. J., \& van Heerden, J. (2004). The Concept of Validity. Psychological Review, 111(4), 1061-1071. https://doi.org/10.1037/0033-295X.111.4.1061

Brennan, R. L. (2005). Generalizability Theory. Educational Measurement: Issues and Practice, 11(4). https://doi.org/10.1111/j.1745-3992.1992.tb00260.x

Campbell, D. T. (1957). Factors relevant to the validity of experiments in social settings. Psychological Bulletin, 54(4), 297-312. https://doi.org/10.1037/h0040950

Chmielewski, M., \& Watson, D. (2009). What is being assessed and why it matters: The impact of transient error on trait research. Journal of Personality and Social Psychology, 97(1). https://doi.org/10.1037/a0015618

Cho, E. (2016). Making Reliability Reliable. Organizational Research Methods, 19(4), 651-682. 


\section{ASSESING THE RELIABILITY OF FORMATIVE INSTRUMENTS}

https://doi.org/10.1177/1094428116656239

Cortina, J. M. (1993). What is coefficient alpha? An examination of theory and applications. Journal of Applied Psychology, 78(1), 98-104. https://doi.org/10.1037/0021-9010.78.1.98

Crocker, L., \& Algina, J. (1986). Introduction to classical and modern test theory. Holt. Rinehart and Winston.

Cronbach, L. J. (1951). Coefficient alpha and the internal structure of tests. Psychometrika, 16(3). https://doi.org/10.1007/BF02310555

Cronbach, L. J., \& Shavelson, R. J. (2004). My Current Thoughts on Coefficient Alpha and Successor Procedures. Educational and Psychological Measurement, 64(3), 391-418. https://doi.org/10.1177/0013164404266386

Cumming, G. (2012). Understanding the new statistics: effect sizes, confidence intervals, and metaanalysis. Routledge Taylor \& Francis Group.

Dalege, J., Borsboom, D., van Harreveld, F., van den Berg, H., Conner, M., \& van der Maas, H. L. J. (2016). Toward a formalized account of attitudes: The Causal Attitude Network (CAN) model. Psychological Review, 123(1), 2-22. https://doi.org/10.1037/a0039802

Dima, A. L. (2018). Scale validation in applied health research: tutorial for a 6-step R-based psychometrics protocol. Health Psychology and Behavioral Medicine, 6(1), 136-161. https://doi.org/10.1080/21642850.2018.1472602

Dunn, T. J., Baguley, T., \& Brunsden, V. (2014). From alpha to omega: A practical solution to the pervasive problem of internal consistency estimation. British Journal of Psychology, 105(3), 399-412. https://doi.org/10.1111/bjop.12046

Edwards, J. R. (2011). The Fallacy of Formative Measurement. Organizational Research Methods, 14(2), 370-388. https://doi.org/10.1177/1094428110378369

Edwards, J. R., \& Bagozzi, R. (2000). On the nature and direction of relationships between constructs and measures. Psychological Methods, 5(2), 155-174. https://doi.org/10.1037//1082989X.5.2.155

Falk, C. F., \& Savalei, V. (2011). The Relationship Between Unstandardized and Standardized Alpha, 
True Reliability, and the Underlying Measurement Model. Journal of Personality Assessment, 93(5), 445-453. https://doi.org/10.1080/00223891.2011.594129

Fishbein, M., \& Ajzen, I. (2010). Predicting and changing behaviour: The reasoned action approach. Taylor and Francis.

Fleuren, B. P. I., van Amelsvoort, L. G. P. M., Zijlstra, F. R. H., de Grip, A., \& Kant, Ij. (2018). Handling the reflective-formative measurement conundrum: a practical illustration based on sustainable employability. Journal of Clinical Epidemiology, 103, 71-81. https://doi.org/10.1016/j.jclinepi.2018.07.007

Fried, E. I. (2017). What are psychological constructs? On the nature and statistical modeling of emotions, intelligence, personality traits and mental disorders. Health Psychology Review, $O(0)$, 1-11. https://doi.org/10.1080/17437199.2017.1306718

Fried, E. I., \& Nesse, R. M. (2015). Depression sum-scores don't add up: why analyzing specific depression symptoms is essential. BMC Medicine, 13(1), 72. https://doi.org/10.1186/s12916-015$0325-4$

Fried, E. I., van Borkulo, C. D., Cramer, A. O. J., Boschloo, L., Schoevers, R. A., \& Borsboom, D. (2017). Mental disorders as networks of problems: a review of recent insights. Social Psychiatry and Psychiatric Epidemiology, 52(1), 1-10. https://doi.org/10.1007/s00127-016-1319-z

Graham, J. M. (2006). Congeneric and (Essentially) Tau-Equivalent Estimates of Score Reliability: What They Are and How to Use Them. Educational and Psychological Measurement, 66(6), 930-944. https://doi.org/10.1177/0013164406288165

Gruijters, S. L. K. (2019). Using principal component analysis to validate psychological scales: Bad statistical habits we should have broken yesterday II. European Health Psychologist, 20(5), 544 549. https://doi.org/10.31234/osf.io/qbe6k

Gruijters, S. L. K., \& Fleuren, B. P. I. (2018). Measuring the Unmeasurable: The Psychometrics of Life History Strategy. Human Nature, 29(1), 33-44. https://doi.org/10.1007/s12110-017-9307-x

Gruijters, S. L. K., \& Peters, G.-J. Y. (2020). Meaningful Change Definitions: Sample size planning for experimental intervention research. Psychology \& Health, In press. 


\section{ASSESING THE RELIABILITY OF FORMATIVE INSTRUMENTS}

https://doi.org/10.1080/08870446.2020.1841762

Guttman, L. (1945). A basis for analyzing test-retest reliability. Psychometrika, 10(4), 255-282. https://doi.org/10.1007/BF02288892

Hardin, A. (2017). A call for theory to support the use of causal-formative indicators: A commentary on Bollen and Diamantopoulos (2017). Psychological Methods, 22(3), 597-604. https://doi.org/10.1037/met0000115

Hardin, A., \& Marcoulides, G. A. (2011). A Commentary on the Use of Formative Measurement. Educational and Psychological Measurement, 71(5), 753-764. https://doi.org/10.1177/0013164411414270

Hayes, A. F., \& Coutts, J. J. (2020). Use Omega Rather than Cronbach's Alpha for Estimating Reliability. But... Communication Methods and Measures, 14(1), 1-24. https://doi.org/10.1080/19312458.2020.1718629

Howell, R. D., Breivik, E., \& Wilcox, J. B. (2007). Reconsidering formative measurement. Psychological Methods, 12(2), 205-218. https://doi.org/10.1037/1082-989X.12.2.205

Hussey, I., \& Hughes, S. (2020). Hidden Invalidity Among 15 Commonly Used Measures in Social and Personality Psychology. Advances in Methods and Practices in Psychological Science, 3(2), 166-184. https://doi.org/10.1177/2515245919882903

Jarvis, C. B., MacKenzie, S. B., \& Podsakoff, P. M. (2003). A Critical Review of Construct Indicators and Measurement Model Misspecification in Marketing and Consumer Research. Journal of Consumer Research, 30(1), 2003. https://doi.org/10.1086/376806

Loken, E., \& Gelman, A. (2017). Measurement error and the replication crisis. Science, 355(6325), 584-585. https://doi.org/10.1126/science.aal3618

MacKenzie, S. B., Podsakoff, P. M., \& Jarvis, C. B. (2005). The Problem of Measurement Model Misspecification in Behavioral and Organizational Research and Some Recommended Solutions. Journal of Applied Psychology, 90(4), 710-730. https://doi.org/10.1037/0021-9010.90.4.710

Maul, A. (2017). Rethinking Traditional Methods of Survey Validation. Measurement: Interdisciplinary Research and Perspectives, 15(2), 51-69. 


\section{ASSESING THE RELIABILITY OF FORMATIVE INSTRUMENTS}

https://doi.org/10.1080/15366367.2017.1348108

McGrath, R. E. (2005). Conceptual Complexity and Construct Validity. Journal of Personality Assessment, 85(2), 112-124. https://doi.org/10.1207/s15327752jpa8502_02

McGraw, K. O., \& Wong, S. P. (1996). Forming inferences about some intraclass correlation coefficients. Psychological Methods, 1(1), 30-46. https://doi.org/10.1037/1082-989X.1.1.30

Nunnally, J. C., \& Bernstein, I. H. (1994). Psychometric Theory. McGraw-Hill, Inc. .

Pearl, J., \& Mackenzie, D. (2018). The Book of Why: The new science of cause and effect. Basic Books.

Perkins, D. O., Wyatt, R. J., \& Bartko, J. J. (2000). Penny-wise and pound-foolish: the impact of measurement error on sample size requirements in clinical trials. Biological Psychiatry, 47(8), 762-766. https://doi.org/10.1016/S0006-3223(00)00837-4

Peters, G.-J. Y. (2014). The alpha and the omega of scale reliability and validity. The European Health Psychologist, 16(2), 56-69.

Phillips, G. W., \& Jiang, T. (2016). Measurement Error and Equating Error in Power Analysis. Practical Assessment, Research \& Evaluation.

Ramos-Villagrasa, P. J., Barrada, J. R., Fernández-del-Río, E., \& Koopmans, L. (2019). Assessing Job Performance Using Brief Self-report Scales: The Case of the Individual Work Performance Questionnaire. Revista de Psicología Del Trabajo y de Las Organizaciones, 35(3), 195-205. https://doi.org/10.5093/jwop2019a21

Revelle, W. (2020). psych: Procedures for Personality and Psychological Research. Version = 2.0.12. https://cran.r-project.org/package=psych

Revelle, W., \& Zinbarg, R. E. (2009). Coefficients alpha, beta, omega, and the glb: Comments on Sijtsma. Psychometrika, 74(1), 145-154.

Rhemtulla, M., van Bork, R., \& Borsboom, D. (2015). Calling Models With Causal Indicators “Measurement Models" Implies More Than They Can Deliver. Measurement: Interdisciplinary Research and Perspectives, 13(1), 59-62. https://doi.org/10.1080/15366367.2015.1016343

Schmidt, F. L., \& Hunter, J. E. (1999). Theory Testing and Measurement Error. Intelligence, 27(3). 


\section{ASSESING THE RELIABILITY OF FORMATIVE INSTRUMENTS}

https://doi.org/10.1016/S0160-2896(99)00024-0

Schmidt, F. L., Le, H., \& Ilies, R. (2003). Beyond alpha: An empirical examination of the effects of different sources of measurement error on reliability estimates for measures of individualdifferences constructs. Psychological Methods, 8(2). https://doi.org/10.1037/1082-989X.8.2.206

Shadish, W. R., Cook, T. D., \& Campbell, D. T. (2002). Experimental and Quasi-Experimental Designs for Generalized Causal Inference. Boston: Houghton Mifflin. In Experimental and QuasiExperimental Designs for Generalized Causal Inference.

Shieh, G. (2016). Choosing the best index for the average score intraclass correlation coefficient. Behavior Research Methods, 48(3), 994-1003. https://doi.org/10.3758/s13428-015-0623-y

Shrout, P. E., \& Fleiss, J. L. (1979). Intraclass correlations: Uses in assessing rater reliability. Psychological Bulletin, 86(2), 420-428. https://doi.org/10.1037/0033-2909.86.2.420

Sijtsma, K. (2009). On the use, the misuse, and the very limited usefulness of Cronbach's alpha. Psychometrika, 74(1), 107-120.

Spector, P. E. (1985). Measurement of human service staff satisfaction: Development of the Job Satisfaction Survey. American Journal of Community Psychology, 13(6), 693-713. https://doi.org/10.1007/BF00929796

Van Bork, R., Wijsen, L. D., \& Rhemtulla, M. (2017). Toward a Causal Interpretation of the Common Factor Model. Disputatio, 9(47), 581-601. https://doi.org/10.1515/disp-2017-0019

Weir, J. P. (2005). Quantifying Test-Retest Reliability Using the Intraclass Correlation Coefficient and the SEM. The Journal of Strength and Conditioning Research, 19(1), 231. https://doi.org/10.1519/15184.1

Wilcox, J. B., Howell, R. D., \& Breivik, E. (2008). Questions about formative measurement. Journal of Business Research, 61(12), 1219-1228. https://doi.org/10.1016/j.jbusres.2008.01.010

Woodward, J. (2003). Making things happen: A theory of causal explanation. Oxford University Press. 
Footnotes.

${ }^{I}$ Further distinctions between types of formative indicators have been suggested, including a distinction between causal and composite indicators. We refrain from further distinguishing between these subtypes because it does not bear significance for our discussion of the internal consistency approach.

${ }^{2}$ Note that this usage of the ICC applies to reflective measurements, where it would for instance be possible to estimate such interactions in line with the CES-method described by Schmidt et al. (2002).

${ }^{3}$ Specifically, this ICC is estimated by a mixed-effects ANOVA, where persons are treated as a random factor and test occasion as a fixed factor. The estimate expresses the reliability of a single measurement.

${ }^{4}$ Note that we explicitly suggesting using the ICC(C,I) for consistency for corrections of attenuation, since the systematic error estimated by the ICC(A,I) does not have an attenuating effect on correlations. 